\title{
MIMO Radar with Broadband waveforms: Smearing Filter Banks and 2D Virtual Arrays
}

\author{
P. P. Vaidyanathan \\ Dept. of Electrical Engineering \\ California Institute of Technology \\ Pasadena, CA 91125 \\ Email: ppvnath@systems.caltech.edu
}

\author{
Piya Pal \\ Dept. of Electrical Engineering \\ California Institute of Technology \\ Pasadena, CA 91125 \\ Email: piyapal@caltech.edu
}

\author{
Chun-Yang Chen \\ Dept. of Electrical Engineering \\ California Institute of Technology \\ Pasadena, CA 91125 \\ Email: cyc@caltech.edu
}

\begin{abstract}
In this paper MIMO radars with broadband waveforms are considered. A time domain viewpoint is taken, which allows frequency invariant beamforming with a filter bank called the smearing filter bank. Motivated by recent work on two dimensional arrays to obtain frequency invariant one dimensional beams, the generation of two dimensional virtual arrays from one dimensional ULAs is also considered. It is also argued that when the smearing filter bank is appropriately used, frequency invariant $2 \mathrm{D}$ beams can be generated.
\end{abstract}

\section{INTRODUCTION}

The concept of MIMO radars has created considerable excitement in recent years [1], [2], [4], [6], [10], [11]. When the transmitted signals in a MIMO radar are broadband, the beamforming performance becomes frequency dependent, and special care should be taken to ensure frequency invariance. A standard technique is to replace the constant beamforming multipliers with filters [15], [3], [8]. For MIMO radars since the virtual array at the receiver can be quite long [1], this results in large complexity at the receiver. A more fundamental issue is that the concept of a virtual array does not extend to the broad band case unless we exercise special care, as we shall see. Broadband issues in MIMO radar have been discussed in the past though not extensively [7].

In this paper we present some new directions for broad band MIMO radar. We first present a time domain view of the effect of broadband signals (Sec. II), and then propose the use of a so-called smearing filter bank at the receiver, to achieve frequency invariance (Sec. III). The method is applicable to SIMO as well as MIMO radars. One of the fundamental ideas in MIMO radars is the generation of the virtual array [1]. In Sec. IV we consider the generation of two dimensional virtual arrays and discuss the issues that arise in the broadband case. This section was motivated by the work in [5] wherein it is shown that frequency invariance can be achieved in one dimensional beamforming by using a $2 \mathrm{D}$ array with constant multipliers rather than filters. But we will find that there are major differnces in Sec. IV.

\footnotetext{
${ }^{1}$ Work supported in parts by the Office of Naval Research grant N00014-08-1-0709, and the California Institute of Technology.
}

\section{BROADBAND SIGNALS IN MIMO RADAR}

In this section we take a fresh look at the effect of wideband signals on beamforming. This opens up a new way to achieve frequency invariance in beamforming. This approach, called the smearing filter bank approach, is described in Sec. III.

\section{II.1 Wideband case, standard frequency domain view}

Given an $N$ element receiving ULA with element spacing $d$, the signal received at the $n$th element from direction of arrival (DOA) $\theta$ (measured from the normal to the ULA) has the form $e^{j \omega_{c} t} e^{-j \omega_{c} n d \sin \theta / c}$ in the narrowband case. Here $c$ represents the speed of propagation and $\omega_{c}$ is the carrier frequency. (The delay due to range is ignored, as it is fixed for a given range cell). After demodulating the carrier we therefore have the signals $e^{-j \omega_{c} n d \sin \theta / c}, 0 \leq n \leq N-1$, which are used in beamforming. In the broadband case the $n$th sensor receives a superposition of these single-frequency components

$$
r_{n}(t)=\int X(j \omega) e^{j \omega t} e^{-j \omega(n d \sin \theta / c)} \frac{d \omega}{2 \pi}
$$

where the transmitted signal $x(t)$ has Fourier transform $X(j \omega)$ supported in a band of width $B \mathrm{~Hz}$ (e.g., $-\pi B<\omega<$ $\pi B)$. The traditional way to obtain a frequency invariant beam is to filter $r_{n}(t)$ with a filter $A_{n}(j \omega)$ and sum the results: $y(t)=\sum_{n=0}^{N-1}\left(a_{n} * r_{n}\right)(t)$, where $*$ denotes convolution. In the frequency domain this takes the form $Y(j \omega)=$ $\sum_{n=0}^{N-1} A_{n}(j \omega) R_{n}(j \omega)$, that is,

$$
Y(j \omega)=X(j \omega) \sum_{n=0}^{N-1} A_{n}(j \omega) e^{-j \omega(n d \sin \theta / c)}
$$

(The notation $Y(j \omega, \theta)$ would be more complete but we keep it simple). The filters $A_{n}(j \omega)$ are designed such that the above summation is almost independent of $\omega$, that is,

$$
\sum_{n=0}^{N-1} A_{n}(j \omega) e^{-j \omega(n d \sin \theta / c)} \approx B(\theta)
$$

The quantity $B(\theta)$ is then the frequency invariant beam pattern. In this case we approximately have

$$
Y(j \omega)=X(j \omega) B(\theta)
$$


or equivalently $y(t)=x(t) B(\theta)$. Thus the original waveform $x(t)$ is reproduced, with the beam pattern's gain $B(\theta)$ attached to it at each angle $\theta$. Many interesting methods have been reported [3], [8], [12], [15] for the design of the filter bank $\left\{A_{n}(j \omega)\right\}$ which achieves the frequency invariance (3).

\section{II.2 Wideband case, time domain view}

If $s(t)$ is the waveform transmitted on a carrier $\omega_{c}$, the received signal at the $n$th sensor is

$$
s\left(t-t_{n}\right) e^{j \omega_{c} t} e^{-j \frac{\omega_{c}}{c}(n d \sin \theta)}
$$

where $t_{n}=n d \sin \theta / c$. We have assumed a point target, and neglected to show the noise and clutter terms; any frequency dependence of the radar cross section is ignored for simplicity. Unlike in the previous subsection we have shown the carrier and baseband waveforms separately. With $d=\lambda / 2$ the delay in the baseband signal $s(n)$ becomes

$$
t_{n}=\frac{n \lambda \sin \theta}{2 c}=\left(\frac{\pi \sin \theta}{\omega_{c}}\right) n
$$

where we have used $c=\omega_{c} \lambda / 2 \pi$. So the delay depends on the direction of arrival $\theta$, the element location $n$, and the carrier frequency $\omega_{c}$. For fixed DOA $\theta$, we see that the delay is a linear progression:

$$
0, \tau_{\theta}, 2 \tau_{\theta}, \ldots,(N-1) \tau_{\theta}
$$

as demonstrated in Fig. 1. The worst possible delay therefore occurs when $\sin \theta=1$ and $n=N-1$, and is given by

$$
\tau_{\max }=\frac{(N-1) \pi}{\omega_{c}}
$$

For negative $\theta, s\left(t-t_{0}\right)$ is delayed with respect to $s(t-$ $t_{N-1}$ ), so the total range in delay is $2 \tau_{\max }$. Now, the significant duration of the baseband waveform $s(t)$ is inversely proportional to its bandwidth $B$. More precisely, the autocorrelation of $s(t)$ (output of the matched filter which is typically used at the receiver) has duration of the order of $1 / B$, and $s(t)$ itself has duration $Q / B$ where $Q$ is the pulse compression ratio. Since the matched filter output is what determines the performance, let us assume the duration of interest is

$$
D=\frac{1}{B}
$$

The question is, how does the worst case delay $\tau_{\max }$ compare with this duration? The delay $\tau_{\max }$ is inversely proportional to the carrier frequency $\omega_{c}$, whereas the duration $D$ is inversely proportional to the bandwidth $B$. In the narrowband case we have $B<<\omega_{c} / 2 \pi$, or equivalently

$$
\frac{\omega_{c}}{2 \pi}>>B
$$

so that

$$
\tau_{\max }<<D \quad \text { (narrowband case), }
$$

and the delays $t_{n}$ are negligible as demonstrated in Fig. 2. So, beamforming can be done under the usual assumption that the matched filter output at the $n$th sensor after sampling is

$$
y(n)=e^{-j \frac{\omega_{c}}{c}(n d \sin \theta)}
$$

In the wideband case, however, these delays are not negligible, as demonstrated in Fig. 1.

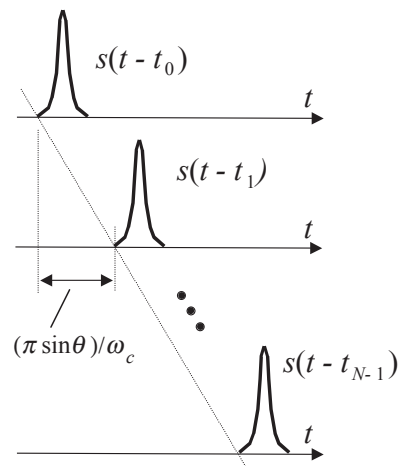

Fig. 1. The received waveform $s\left(t-t_{n}\right)$ at the $N$ sensor locations, in the wideband case.

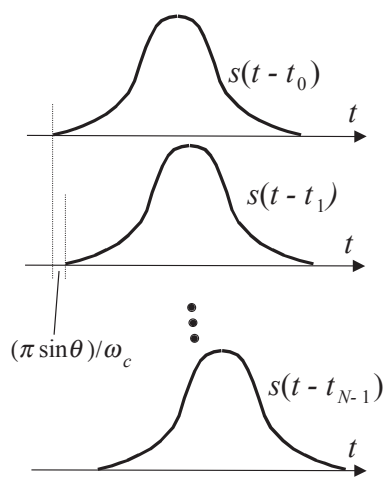

Fig. 2. The received waveform $s\left(t-t_{n}\right)$ at the $N$ sensor locations, in the narrow band case.

For the case of MIMO radars the preceding discussions continue to hold, but there are important differences. Here the transmitter is an ULA with $M$ elements and the receiver is a (colocated) ULA with $N$ elements. The signal $s_{m}(t)$ transmitted by the $m$ th transmitting element is received at the $n$th receiving array element in the form

$$
y_{m, n}(t)=s_{m}\left(t-t_{T, m}-t_{R, n}\right) e^{-j \omega_{c}\left(t_{T, m}+t_{R, n}\right)}
$$

where we have assumed that the carrier $e^{j \omega_{c} t}$ is removed by demodulation. Here the delays $t_{T, m}$ and $t_{R, n}$ are

$$
t_{T, m}=\frac{m d_{T} \sin \theta}{c}, \quad t_{R, n}=\frac{n d_{R} \sin \theta}{c} .
$$

Substituting the typical values $d_{R}=\lambda / 2$ and $d_{T}=N d_{R}$, the worst case delay is

$$
\tau_{\max }=\frac{(M N-1) \pi}{\omega_{c}}
$$

Once again, this cannot be neglected in the broadband case. The broad band effect is more pronounced in the MIMO 
radar, because of the presence of $M N$ instead of $N$. Notice that in the narrowband case $s_{m}\left(t-t_{T, m}-t_{R, n}\right)$ in Eq. (12) can be replaced with $s_{m}(t)$. After using a matched filter bank $\left\{H_{k}(j \omega)\right\}$ at the output of each sensor (Fig. 3), we can then form a virtual array with $M N$ elements because of the phases of the terms $e^{-j \omega_{c}\left(t_{T, m}+t_{R, n}\right)}$. In the broadband case however, because of the presence of $s_{m}\left(t-t_{T, m}-t_{R, n}\right)$, we do not have a virtual array yet. We have to work a little harder for that.

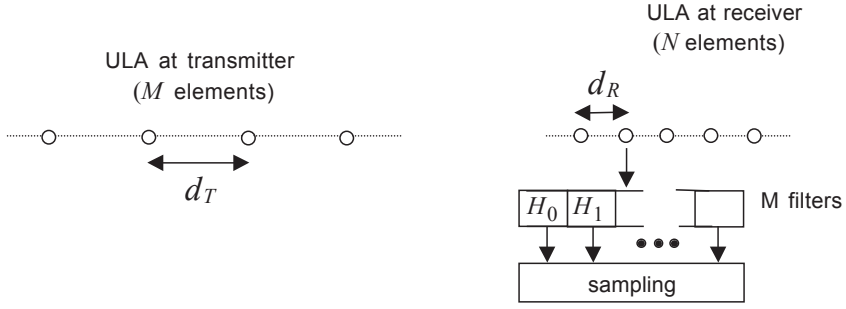

Fig. 3. The conventional MIMO radar with $M$ transmit antenna elements and $N$ receiving antenna elements.

\section{THE SMEARING FILTER BANK FOR FREQUENCY INVARIANCE}

We see therefore that in the broadband case the outputs of the matched filters have extra shifts which depend on the DOA, the element location, and the bandwidth (in relation to carrier). This is true for SIMO as well as MIMO radars. The difference in the MIMO case is that $N$ is replaced with $M N$ and $t_{n}$ becomes doubly indexed $\left(t_{m, n}=t_{T, m}+t_{R, n}\right)$.

For digital beamforming, we have to sample the matched filter outputs at the peak, and this becomes difficult because of the delays $t_{n}$ which unfortunately depend on the DOA $\theta$. A simple way to overcome this difficulty is to smear the narrow pulses at the matched filter outputs before sampling: we smear the pulses by a certain amount so that all of the delayed waveforms will have identical magnitudes at a certain common point $t_{s}$ in time as demonstrated in Fig. 4. (In practice, we would have one such sampling instant $t_{s}$ in each range cell.) This is quite practicable since the worst-case delay (7) is known. The tradeoff is that the sharpness of the matched filters is compromised, and so is the SNR because matched filters maximize the SNR. However, the frequency dependence of beamforming is completely eliminated.

It is true that smearing reduces the range resolution because the sharpness of the pulse is compromised. Buf if this is not done, then the potentiality for range resolution arising from sharpness of pulses is not useful anyway (because the received pulses are staggered). The total widening or smearing of the pulse is equal to the worst case delay suffered due to broadband property.

The smearing can be achieved by using a simple filter whose impulse reponse approximates a flat pulse. In fact this filter need not be the same for all the pulses. From Fig. 1 we see that the first pulse has to be smeared the most. So we can have a smearing filter bank with the amount of smearing minimized according to need. In fact one can seek to find the optimal smearing filter bank which also takes SNR into account.

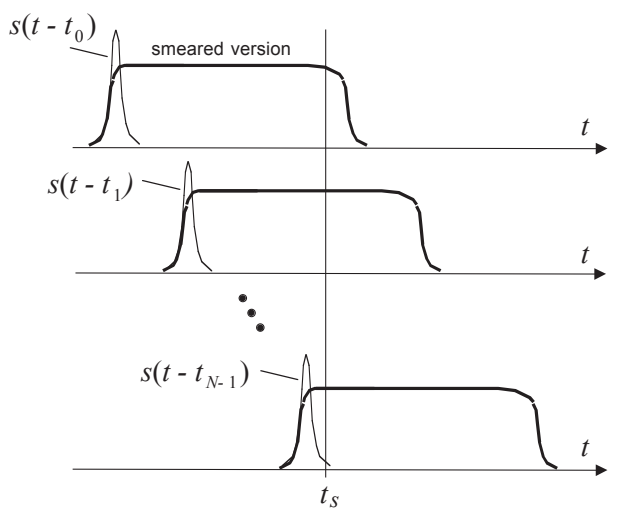

Fig. 4. Smearing the matched filter outputs before sampling.

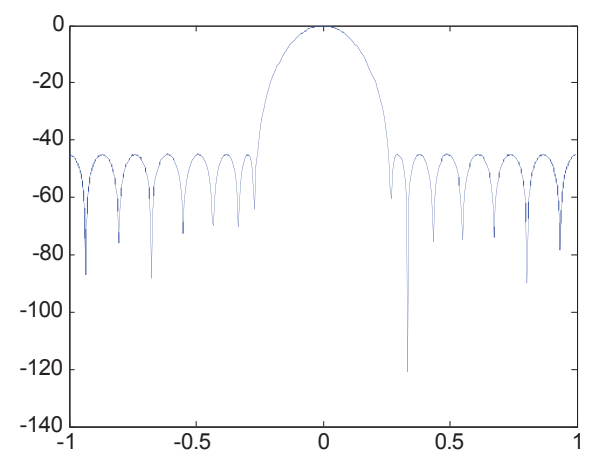

Fig. 5. Beam pattern after pulse smearing.

Since each matched filter can be combined with its smearing filter in cascade, the problem reduces to one of optimizing a filter bank

$$
G_{k}(j \omega), 0 \leq k \leq N-1
$$

at the output of each of the $N$ receiving sensors. The smearing filters are followed by uniform samplers, after which we can do digital beamforming as usual. Figure 5 shows an example of a Dolph-Chebyshev beam generated in this way. In this example the waveform $s(t)$ is a broad band chirp, the array has 15 antenna elements, and the smearing filters are simple, identical, lowpass filters.

For the MIMO radar the filter bank has to be implemented in two stages, like a tree sructure. First we use a smearing filter $G_{k}(j \omega)$ at the output of each sensor to compensate for $t_{R, n}$. Then we pass each of these outputs through a smearing filter bank $\left\{H_{k}(j \omega)\right\}$ to compensate for $t_{T, n}$. The outputs of these $M N$ filters are sampled at a fixed time in each range cell. We can then form a virtual array at the receiver. Notice 
that in the MIMO radar case, in order to avoid cross talk between the $M$ signals after matched filtering, the orthogonality condition $\int_{-\infty}^{\infty} s_{m}(t) s_{k}^{*}(t) d t=\delta(k-m)$ has to be replaced with the stronger condition $\int_{-\infty}^{\infty} s_{m}(t) s_{k}^{*}(t-\tau) d t=$ $\delta(k-m)$, for all $\tau$. This is equivalent to $S_{m}(j \omega) S_{k}^{*}(j \omega)=0$ for all $\omega$ (non overlapping frequency bands). Such waveforms can be approximated using uniform filter banks with good prototype lowpass filters [13].

\section{VIRTUAL ARRAYS IN HIGHER DIMENSIONS}

It has recently been shown by Ghavami [5] that frequency invariance can be achieved in one dimensional beamforming by using a $2 \mathrm{D}$ array with constant multipliers rather than filters. (In this application the 2D array performs beamforming only in the azimuth dimension.) We first explain this and then consider the generation of 2D virtual arrays from 1D linear arrays. Thus, first consider the 2D uniform array shown in Fig. 6. If a plane wave arrives at elevation $\phi$ and azimuth $\theta$, then the path difference between the 0th element and the $(m, n)$ th element is given by $\left(m d_{x} \cos \theta+n d_{y} \sin \theta\right) \sin \phi$. Here $\sin \phi$ is due to the projection of the path difference onto the $X Y$-plane, and $\cos \theta$ and $\sin \theta$ arise due to further projections along the $X$ and $Y$ axes. Since a path difference of $d$ corresponds to a time difference of $d / c$ where $c$ is the velocity of wave propagation, the signal arriving at the $(m, n)$ th sensor element is proportional to

$$
e^{j \omega_{c} t} e^{-j \frac{\omega_{c}}{c}\left(m d_{x} \cos \theta+n d_{y} \sin \theta\right) \sin \phi}
$$

This holds in the narrowband case. In the broadband case, this is replaced with a superposition of frequencies:

$$
y_{m, n}(t)=\int X(j \omega) e^{j \omega t} e^{-j \frac{\omega}{c}\left(m d_{x} \cos \theta+n d_{y} \sin \theta\right) \sin \phi} \frac{d \omega}{2 \pi}
$$

or equivalently

$$
Y_{m, n}(j \omega)=X(j \omega) e^{-j \frac{\omega}{c}\left(m d_{x} \cos \theta+n d_{y} \sin \theta\right) \sin \phi}
$$

To see how the $2 \mathrm{D}$ array can be used for one dimensional frequency invariant beam forming, set $\phi=\pi / 2$. If we use constant multipliers $\alpha_{m, n}$ for beamforming, the beamformer gain is

$$
\sum_{n=0}^{N-1} e^{\frac{-j \omega\left(n d_{y} \sin \theta\right)}{c}} \sum_{m=0}^{M-1} \alpha_{m, n} e^{\frac{-j \omega\left(m d_{x} \cos \theta\right)}{c}}
$$

The doubly indexed multipliers $\alpha_{m, n}$ offer the freedom to force the preceding expression to be approximately independent of $\omega$. Thus, instead of having a singly indexed set of filters as in earlier work, there is a doubly indexed set of numbers in [5] to achieve frequency invariance. For design details see [5].

\section{IV.1 2D Virtual arrays}

Now consider Fig. 7 which shows a MIMO radar system with a one dimensional uniform linear array with $M$ elements at the transmitter, and a one dimensional uniform linear array with $N$ sensor elements at the receiver. The arrays are in orthogonal directions. The $m$ th transmitting element transmits the waveform $s_{m}(t)$ on a common carrier frequency $\omega_{c}$.

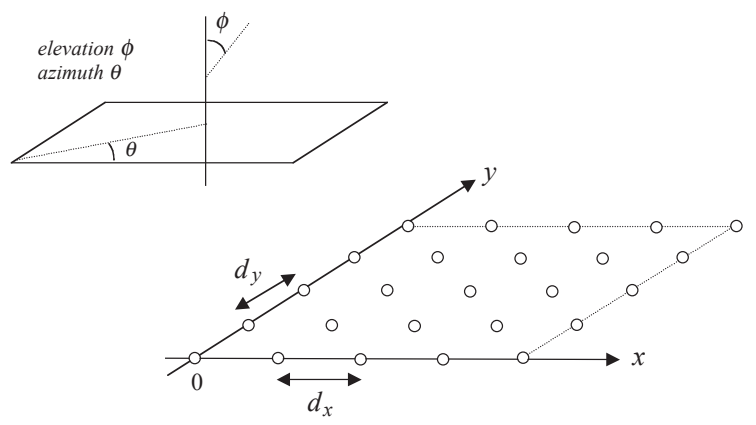

Fig. 6. A two dimensional uniform sensor array.

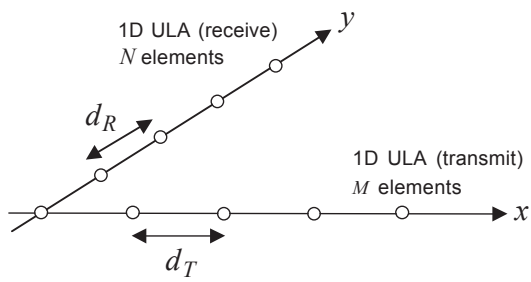

Fig. 7. A MIMO radar system with one dimensional orthogonal arrays at transmitter and receiver.

Assuming that the transmitter and receiver are colocated, the signal arriving at the $n$th receiving element is a superposition of the waveforms transmitted from the $M$ transmitting elements, and is given by

$\sum_{m=0}^{M-1} s_{m}\left(t-t_{T, m}-t_{R, n}\right) e^{j \omega_{c} t} e^{-j \frac{\omega_{c}}{c}\left(m d_{T} \cos \theta+n d_{R} \sin \theta\right) \sin \phi}$

Here the delays $t_{T, m}$ and $t_{R, n}$ are created due to the locations of the $m$ th transmitting and $n$th receiving elements, and are given by

$$
t_{T, m}=\frac{m d_{T} \cos \theta \sin \phi}{c}, \quad t_{R, n}=\frac{n d_{R} \sin \theta \sin \phi}{c}
$$

First consider the narrowband case (bandwidth of $s_{m}(t)$ is $<<\omega_{c}$ ) where we can neglect the effect of delays to write:

$$
s_{m}\left(t-t_{T, m}-t_{R, n}\right) \approx s_{m}(t)
$$

Thus, after demodulation (multiplication with $e^{-j \omega_{c} t}$ ) the signal at the $n$th receiving sensor is

$$
r_{n}(t)=\sum_{m=0}^{M-1} s_{m}(t) e^{-j \frac{\omega_{c}}{c}\left(m d_{T} \cos \theta+n d_{R} \sin \theta\right) \sin \phi}
$$

This signal is filtered by a matched filter bank with $M$ filters $h_{k}(t)=s_{k}^{*}(-t)$. Since the transmitted waveforms are usually 
orthogonal:

$$
\int_{-\infty}^{\infty} s_{m}(t) s_{k}^{*}(t) d t=\delta(k-m)
$$

the output of the $m$ th matched filter connected to the $n$th receiveing sensor is given by

$$
y(m, n)=e^{-j \frac{\omega_{c}}{c}\left(m d_{T} \cos \theta+n d_{R} \sin \theta\right) \sin \phi}
$$

Note that this is the matched filter output sampled at zero time where the autocorrelation of $s_{m}(t)$ has its peak. This is precisely the signal received by the 2D array (Eq. (16)). Thus the narrowband MIMO radar with two 1D ULAs produces a 2D virtual array with element spacings $d_{T}$ and $d_{R}$ along the horizontal and vertical directions. This idea can be extended to other geometries. For example consider Fig. 8. This shows a MIMO radar system with a 1D uniform linear array at the transmitter, and a circular array at the receiver (colocated). The plane of the circular array is perpendicular to the transmitting ULA. Under the narrow band assumption, we obtain a cylindrical virtual array in three dimensions as shown in the figure.

In the broad band case, we have to use smearing filter banks before sampling, in order to be able to obtain a virtual array, as described in Sec. III. By using this technique, we can therefore obtain two and higher dimensional virtual arrays of the kind shown in the figure. Since these arrays can be rendered frequency invariant by the use of smearing filters, they can perform two dimensional beamforming unlike [5], where the $2 \mathrm{D}$ array is used to perform frequency invariant beamforming in only one dimension. An altogether different technique to exploit the virtual array concept in the broadband case is reported in [9] which uses orthogonal waveforms $s_{m}(t)$ in different frequency bands. In this case there is no need for the smearing filters.

\section{CONCLUding REMARKS}

We considered a number of interesting problems relating to broad band beamforming and virtual array generation in MIMO radars. The method based on smearing filter banks offers great simplicity and allows us to form MIMO virtual arrays in the broadband case. The smearing method can be related to the DFT method for broad band beamforming [14]; since the DFT system can be considered as a filter bank, retaining the lowpass band is qualitatively similar to the above method. But the above viewpoint shows that there is more freedom in choice of the smearing filters.

The issue of optimizing the smearing filter bank (based on practical criteria such as the SINR) appears to be an interesting problem. In fact, joint optimization of the MIMO radar waveforms $s_{k}(t)$ and the receiving filter bank responses $h_{k}(t)$ is another interesting problem to consider. The viewpoint taken in this paper is that the wideband property is a nuisance which needs to be overcome before beamforming; so only a narrow region of the band is retained in the beamforming. If the wideband signals are information bearing, then the approach in [9] is more appropriate.

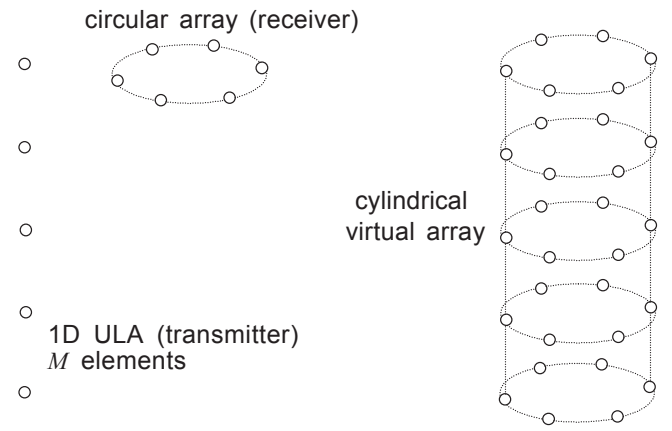

Fig. 8. MIMO radar with a 1D ULA at the transmitter, and a circular array at the receiver. The virtual array is a cylinder.

\section{REFERENCES}

[1] D. W. Bliss and K. W. Forsythe, "Multiple-input multiple-output radar and imaging: degrees of freedom and resolution," 37th Asilomar Conf. on Sig., Sys., and Comp., pp. 54-59, Nov. 2003.

[2] Chun-Yang Chen and P. P. Vaidyanathan, "MIMO radar spacetime adaptive processing using prolate spheroidal wave functions", IEEE Trans. Signal Processing, pp. 623-635, Feb., 2008.

[3] T. Chou, "Frequency-independent beamformer with low response error”, pp. 2995-2998, Proc. of the ICASSP, Detroit, 1995.

[4] E. Fishler, A. Haimovich, R. S. Blum, L. J. Cimini, D. Chizhik, and R. A. Valenzuela, "Performance of MIMO radar systems: Advantages of angular diversity," in Proc. IEEE Asilomar Conf. Sig., Sys., and Comp., pp. 305-309, Nov. 2004.

[5] M. Ghavami, "Wideband smart antenna theory using rectangular array structures," IEEE Trans. Sig. Proc., Sept. 2002.

[6] J. Li, and P. Stoica, "MIMO Radar with Colocated Antennas", IEEE Sig. Proc. Mag., pp. 106-114, Sept. 2007.

[7] B. Liu, C. Han, and B. Liu, "Receiving Signal Processing of Wideband MIMO Radar Based On Transmitting Diversity," Proc. Intl. Conf. on Radar, 2006, pp. 1-4, Oct. 2006.

[8] W. Liu and S. Weiss, "A new class of broadband arrays with frequency invariant beam patterns," pp. II-185 to II-188, Proc. of the ICASSP, Montreal, 2004.

[9] Piya Pal and P. P. Vaidyanathan, "Frequency invariant MVDR beamforming without filters and implementation using MIMO radar", submitted to ICASSP 2009.

[10] F. C. Robey, S. Coutts, D. Weikle, J. C. McHarg, and K. Cuomo, "MIMO Radar Theory and Experimental Results," 38th Asilomar Conf. on Sig., Sys., and Comp., pp. 300-304, Nov. 2004.

[11] G. San Antonio and D. R. Fuhrmann, "Beampattern synthesis for wideband MIMO radar systems," 21st IEEE Intl. Workshop on Comp. Adv. in multisensor adap. proc., Dec. 2005.

[12] T. Sekiguchi, and Y. Karasawa, "Wideband beamspace adaptive array utilizing FIR fan filters for multibeam forming," IEEE Trans. Sig. Proc., pp. 277-284, Jan. 2000.

[13] P. P. Vaidyanathan, Multirate systems and filter banks, Prentice Hall, Inc., 1993.

[14] H. L. Van Trees, Optimum array processing, WileyInterscience, 2002.

[15] D. B., Ward, R. A. Kennedy, and R. C. Williamson, "Theory and design of broadband sensor arrays with frequency invariant far-field beam patterns", J. Acoust. Soc. of America, pp. 10231034, Feb. 1995. 